\title{
Particle size selection device for use with the Turbohaler
}

\author{
Mark L Everard, Sunalene G Devadason, Peter N Le Souef
}

\begin{abstract}
Background - Drug deposited within the upper airways of patients using dry powder inhalers does not contribute to the therapeutic effect but can result in unwanted local side effects and, when swallowed, may contribute to systemic effects. A chamber has been devised which uses the centrifugal force generated by the Turbohaler to remove large "non-respirable" particles with a view to minimising deposition in the upper airway. An in vitro study was performed to determine whether such a chamber could reduce the dose contained in coarse particles without having a significant effect on the "respirable dose". Methods - The mouthpiece of a $200 \mu \mathrm{g}$ Turbohaler was modified to allow a small volume chamber to be attached. The particle size distribution generated by the Turbohaler was assessed using a multistage liquid impinger with a flow rate of $601 / \mathrm{min}$. The quantity of drug on each stage was quantified using an ultraviolet spectrophotometric technique. For each experiment 10 actuations were used to ensure adequate quantities of drug on each stage. Particles depositing on stages $3+$ 4 have a diameter of $<6.8 \mu \mathrm{m}$ and are arbitrarily referred to as the "respirable dose". The particle size distribution obtained using the Turbohaler $(n=10)$ was compared with that from the Turbohaler + chamber $(n=11)$.
\end{abstract}

Results - The addition of the chamber resulted in the mean (SD) dose contained in larger "non-respirable" particles depositing on stages $1+2$ being reduced from $52 \cdot 2(12 \cdot 3)$ to $29.6(6 \cdot 9) \mu \mathrm{g}$ per actuation. However, the chamber did not affect the "respirable" dose. The dose contained in particles with a diameter of $<6.8 \mu \mathrm{m}$ from the standard Turbohaler was $91 \cdot 1$ (8.9) $\mu$ g compared with $82 \cdot 4(18 \cdot 6) \mu \mathrm{g}$ when used with the chamber.

Conclusions - These results indicate that it is possible to devise an effective particle size selection device for the Turbohaler. It may be possible to produce such devices for other dry powder inhalers, although the design would need to be tailored to each particular device.

(Thorax 1996;51:537-539)

Keywords: dry powder inhaler, auxiliary chamber, particle size selection.
Dry powder inhalers are widely used for generating aerosols for the treatment of asthma. Current devices utilise energy provided by the inspiratory effort of the patient to disaggregate the micronised particles. The greater the inspiratory effort, the more effective the disaggregation and the greater the "respirable dose". The improved disaggregation that occurs as inspiratory flows increase more than offsets the increased loss of particles in the upper airways due to the higher velocity and results in improved drug delivery to the lungs. ${ }^{1-8}$ However, even when current dry powder inhalers are used optimally, it is inevitable that a substantial portion of the dose deposits in the upper airways. ${ }^{7-9}$ For bronchodilators this is of little consequence, but for steroids this dose may contribute significantly to both systemic and local side effects.

Spacers and holding chambers are widely used when steroids are delivered via metered dose inhalers in order to reduce the oral pharyngeal dose and so minimise the potential for unwanted side effects. No such device is currently available for dry powder inhalers and therefore a chamber was devised which utilises the centrifugal force generated by the Turbohaler to remove large "non-respirable" particles. The effectiveness of the chamber was determined in vitro using a multistage liquid impinger.

\section{Methods}

As we were unable to manufacture our own chamber, we modified an existing small volume spacer, the ACE chamber (DHD, Canastota, USA), designed for use with metered dose inhalers. This chamber has the general shape we desired but is rather larger than we would have chosen. The chamber and the mouthpiece of a Turbohaler were modified to permit the chamber to be attached to it (figure). Modification of the Turbohaler involved cutting back the mouthpiece to reveal the insert containing the spiral channels; this was then enclosed in a rubber sleeve which could be inserted into the tapered end of the chamber. The chamber was modified by removing the port used for actuating the metered dose inhaler.

An Astra multistage liquid impinger (Copley, Nottingham, UK) was used to assess the total dose and particle size distribution of the aerosol delivered by a $200 \mu \mathrm{g}$ budesonide Turbohaler with $(n=11)$ and without $(n=10)$ the chamber. The multistage liquid impinger operates

Received 9 March 1995

Returned to authors

1 August 1995

Revised version receive

13 December 1995

Accepted for publicatio 


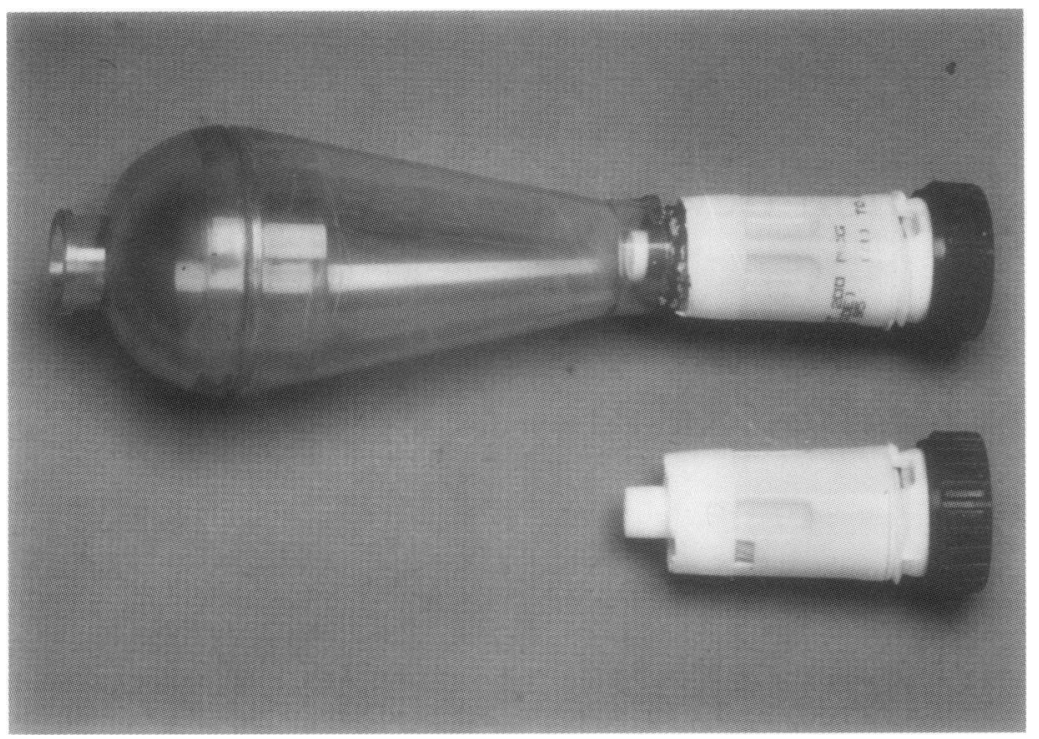

Turbohaler with mouthpiece cut back to reveal the insert containing the disaggregation spirals and a modified Turbohaler attached to a modified ACE spacer device as used in these experiments. (C) Gloucester Royal NHS Trust, 1995.

Mean (SD) dose of budesonide ( $\mu g$ ) per actuation deposited on each stage of the multistage liquid impinger when delivered via the Turbohaler + chamber compared with the Turbohaler alone

\begin{tabular}{lrl}
\hline & $\begin{array}{l}\text { Turbohaler } \\
+ \text { chamber }\end{array}$ & $\begin{array}{l}\text { Standard } \\
\text { Turbohaler }\end{array}$ \\
\hline Stage 1 & $24 \cdot 0(5 \cdot 9)$ & $45 \cdot 5(10 \cdot 1)^{*}$ \\
Stage 2 & $5 \cdot 6(2 \cdot 1)$ & $6 \cdot 7(5 \cdot 6)$ \\
Stage 3 & $21 \cdot 0(4 \cdot 2)$ & $22 \cdot 0(3 \cdot 4)$ \\
Stage 4 & $61 \cdot 4(15 \cdot 2)$ & $69 \cdot 1 \quad(6 \cdot 1)$ \\
\hline${ }^{*}$ p $<0.001$. & &
\end{tabular}

at a flow of $60 \mathrm{l} / \mathrm{min}$ and has four stages, the fourth being an absolute filter. The cut offs for the four stages are $>13 \mu \mathrm{m}$ (stage 1 ), 6.8-13 $\mu \mathrm{m}$ (stage 2), 3.1-6.8 $\mu \mathrm{m}$ (stage 3 ), and $<3 \cdot 1 \mu \mathrm{m}$ (stage 4). Particles reaching stages 3 and 4 are often referred to as "respirable" particles with aerodynamic diameters of $<6.8 \mu \mathrm{m}$.

Ten actuations were used for each experiment to ensure that sufficient quantities of drug were deposited on each stage of the multistage liquid impinger to permit accurate quantification. The amount of budesonide deposited on the mouthpiece and each of the stages of the multistage liquid impinger was measured using an ultraviolet spectrophotometric method. The mouthpiece and stages of the multistage liquid impinger were washed with approximately $40 \mathrm{ml}$ ethanol. The volume of each wash was then made up to a total of $50 \mathrm{ml}$ with ethanol. The absorbance $(\lambda=243 \mathrm{~nm})$ of each sample was measured in duplicate on a spectrophotometer (Hitachi U-2000). The concentration of budesonide in each of the washes was calculated using the absorbance of a solution containing a known concentration of budesonide. The standard curve for budesonide was linear $\left(r^{2}=1.00\right)$ for concentrations between 0 and $30 \mu \mathrm{g} / \mathrm{ml}$.

\section{Results}

The mean (SD) doses of drug deposited on each stage from the Turbohaler, with and without the chamber, are shown in the table.

\section{Discussion}

Results from this study suggest that a substantial reduction in the coarse "non-respirable" fraction of an aerosol generated by the Turbohaler can be achieved by using a simple, appropriately designed chamber. The dose contained in fine "respirable" particles was unaffected, which suggests that a chamber such as this one might significantly reduce the dose depositing in the upper airway without reducing the dose to the lungs.

Disaggregation of the micronised powder delivered from a Turbohaler is enhanced by passage of powder through the spiral mouthpiece. ${ }^{10}$ The spheronised powder is abraded as it is forced over the outer surface of the spiral. As the particles leave the mouthpiece they retain both forward and centrifugal momentum, and hence tend to spiral outwards as they leave the mouthpiece. The chamber operates as a size selection device by initially allowing the plume of powder to expand. The particles are then required to change direction in order to escape via the mouthpiece. The larger particles impact because of their greater inertia, while smaller particles are entrained by the airflow through the mouthpiece and are available to the patient.

Aerosol exiting the chamber will have lost the initial centrifugal momentum and this may further reduce oral deposition by reducing drug deposition resulting from particles impacting on, and adhering to, the moist mucous membranes of the mouth as a result of their tendency to spiral outwards. It is probable that some fine particles which would normally pass straight through the upper airway at a given inspiratory flow are lost in this manner.

These studies were performed using a flow rate of $60 \mathrm{l} / \mathrm{min}$. It is likely that such a device will be less efficient at lower rates because of the lower velocity of particles, although the exact effect is unpredictable as there will be more large particles with high inertia.

It is noticeable that the variability in the dose that is deposited on stages $3+4$ when using the chamber is considerably greater than for the standard device alone. This may be the result of the variable static charge carried by the chamber as has been described for holding chambers, ${ }^{11}$ and this potential problem would need to be addressed if such a chamber were to be used therapeutically.

The ACE chamber was used because it was available. Analysis of slow motion video film of Turbohalers being actuated suggests that a smaller chamber with a slightly different design might be more efficient at removing "nonrespirable" particles. Hence, further work is required to define the optimal size and design for such a particle selective device. However, these results show that it is possible to develop simple effective particle size selection devices for at least some dry powder inhalers.

1 Groth S, Dirken H. Optimal inhalation procedure for the fenoterol powder inhaler. Eur $\mathcal{F}$ Respir Dis 1983;64(Supp 130):17-24.

2 Pedersen S. How to use a Rotahaler. Arch Dis Child 1986 61:11-4.

3 Auty RM, Brown K, Neale M, Snashall PD. Respiratory tract deposition of sodium cromogylcate is highly dependent upon technique of inhalation using the Spinhaler. Br $\mathcal{F}$ Dis Chest 1987;81:371-80. 
4 Hansen $\mathrm{O}$, Pedersen S. Optimal inhalation technique with terbutaline Turbuhaler. Eur f Respir Dis 1989;2:637-9.

5 Pedersen S, Hansen OR, Fuglsang G. Influence of inspiratory flow rate upon the effect of a Turbuhaler. Arch Dis Child 1990;65:308-19.

6 Engel T, Scharling B, Skovsted B, Heinig JH. Effects, side effects and plasma concentrations of terbutaline in adult asthmatics after inhaling from a dry powder inhaler device asthmatics after inhaling from a dry powder inhaler device at different inhalations and

7 Newman SP, Hollingworth A, Clark AR. Effect of different modes of inhalation on drug delivery from a dry powder inhaler. Int $\mathcal{F}$ Pharm 1994;102:127-32.

8 Borgstrom L, Bondesson E, Moren F, Newman SP. Lung deposition of budesonide inhaled via Turbuhaler. A com- parison with terbutaline sulphate. Eur Respir $\mathcal{F}$ 1994;7: 69-73.

9 Borgstrom L, Newman SP, Weisz A, Moren F. Pulmonary deposition of inhaled terbutaline: comparison of scanning gamma camera and urinary excretion methods. 7 Pharm Sci 1992;81:753-5.

10 Jaegfeld $\mathrm{H}$, Andersoon JAR, Trofast E, Wetterlin KIL. Particle size distribution from different modifications of Turbuhaler. In: Newman SP, Moren F, Crompton GK, eds. A new concept in inhalation therapy.

11 O'Callaghan C, Lynch J, Robertson C. Improvement in sodium cromoglycate delivery from a spacer device by use of an antistatic lining, immediate inhalation, and avoiding multiple actuations of drug. Thorax 1993;48:603-6.

\title{
Radiographic features of staphylococcal pneumonia in adults and children
}

\author{
John Macfarlane, Donald Rose
}

\begin{abstract}
Background - Clinical and laboratory features do not accurately correlate with the cause of community acquired pneumonia. A study was performed to examine whether the radiographic features of staphylococcal pneumonia are sufficiently distinct to aid early diagnosis.

Methods - The chest radiographs of 34 patients (including eight children) with proven staphylococcal pneumonia were reviewed by two experienced observers using methods described previously. Features on presentation and follow up were noted.

Results - The most striking features were the presence of multilobar consolidation on presentation, cavitation, pneumatocoeles and spontaneous pneumothorax, together with a tendency to radiographic deterioration after admission in both adults and children. Some of these features are much less common with other causes of community acquired pneumonia. However, most of the cases did not have these classic features.

Conclusions - The presence of certain radiographic features, including multilobar shadowing, cavitation, pneumatocoeles, and spontaneous pneumothorax, are seen with staphylococcal pneumonia in adults and children, but their absence does not exclude the diagnosis.

(Thorax 1996;51:539-540)
\end{abstract}

Keywords: staphylococcal pneumonia, radiographic changes, adults, children, diagnosis.

Staphylococcal pneumonia is an uncommon but serious infection which accounts for about $1 \%$ of community acquired pneumonias. ${ }^{1}$ The clinical and laboratory features of different forms of community acquired infection have been shown not to be an accurate predictor of aetiology. ${ }^{2}$ Staphylococcal pneumonia is said to have characteristic radiographic features ${ }^{34}$ and, as such, these may assist early clinical identification of staphylococcal infection to allow appropriate antibiotic therapy. However, no studies have been made of the radiographic appearances of staphylococcal pneumonia since effective antibiotics became widely available which may act to modify the radiographic picture of the infection. We have previously reported on the clinical and laboratory features of 61 cases of community acquired staphylococcal pneumonia in adults ${ }^{5}$ and report here the radiographic findings of 34 patients, including eight children, with proven staphylococcal pneumonia.

\section{Methods}

Cases of community acquired staphylococcal pneumonia were identified from admission diagnosis records of the Trent Region and chest radiographs of 34 patients were available for study. As in our previous clinical study, ${ }^{5}$ the diagnosis was accepted if Staphylococcus aureus was isolated as a heavy growth from sputum, tracheal aspirate or from blood, pleural fluid, or necropsy lung tissue.

Available chest radiographs were reviewed by two experienced observers. Lateral views were available in many cases but infrequently provided extra information. Shadowing was recorded as mainly homogeneous or mainly patchy. Other features including pleural fluid, lobar or segmental collapse (loss of volume), cavitation and pneumatocoeles were noted. A pneumatocoele was differentiated from cavitation or abscess formation by the presence of a smooth and thin walled cystic structure, sometimes with a fluid level. A swollen lobe was recognised by an increase in the volume of the lobe, together with a displaced convex fissure. 